\title{
Calpain I Activation Is Specifically Related to Excitatory Amino Acid Induction of Hippocampal Damage
}

\author{
Robert Siman, J. Carl Noszek, and Claire Kegerise \\ Neuroscience Group, The DuPont Company, Wilmington, Delaware 19898
}

\begin{abstract}
Sustained stimulation of receptors for excitatory amino acids leads to both activation of the calcium-dependent cysteine protease calpain I and to the death of receptive neurons. Here, we have examined the relationship between the calpain I activation and neurodegeneration. Calpain I activation was manifested as increased levels of the major proteolytic fragments of the calpain subsirate spectrin, detected and quantified by immunoblotting. Intraventricular administration of the excitatory amino acids kainate or $\mathrm{N}$-methyl-D-aspartate (NMDA) produced calpain I-mediated spectrin degradation and hippocampal neuronal loss. The NMDA antagonist 3-(2-carboxypiperazin-4-yl)-propyl-1-phosphonic acid selectively blocked NMDA- but not kainate-induced protease activation and hippocampal damage. Temporally, spectrin degradation preceded the onset of pyramidal cell degeneration monitored by silver-impregnation histochemistry. Only those doses of kainate $(0.15-1 \mu \mathrm{g})$ or NMDA (40$80 \mu \mathrm{g})$ sufficient to cause hippocampal damage markedly increased spectrin breakdown. Both the neuronal damage and calpain I activation induced by kainate occurred primarily in area CA3. Degeneration of hippocampal neurons evoked by colchicine was not accompanied by calpain activation, indicating that proteolysis is not stimulated simply as a secondary response to neuronal destruction. Thus, a close correspondence exists between excitatory amino acid induction of neuronal degeneratlon and of calpain I-mediated spectrin degradation. The results suggest that calpain 1 may be an intracellular mediator of excitatory amino acid action, and further, they support the hypothesis that calcium influx and calpain I activation are obligatory events in the initiation of excitatory amino acid neurotoxicity.
\end{abstract}

As the major excitatory neurotransmitters in brain, acidic amino acids such as L-glutamate participate in a variety of functions. In addition to their prominent role in moment-to-moment synaptic communication, excitatory amino acids (EAAs) have been implicated in long-lasting physiological and pathological modifications of neurons. EAA activation at one glutamate receptor subtype, that preferentially stimulated by $N$-methyl-D-aspartate (NMDA), is required for the induction of long-term potentiation of synaptic transmission (Collingridge et al., 1983; Harris et al.,

\footnotetext{
Received July 28, 1988; revised Sept. 16, 1988; accepted Sept. 27, 1988.

We wish to thank Janice Lancaster for excellent technical assistance and Dr. Robert B. Nelson for critically reviewing the manuscript.

Correspondence should be addressed to Robert Siman, Neuroscience Group, The DuPont Company, E400/3430, Wilmington, DE 19898.

Copyright (C) 1989 Society for Neuroscience $0270-6474 / 89 / 051579-12 \$ 02.00 / 0$
}

1984; Artola and Singer, 1987) and for establishment of ocular dominance columns in developing visual cortex (Kleinschmidt et al., 1987). Moreover, sustained NMDA receptor activation may have pathological consequences. In animal and in vitro models of stroke (Rothman, 1984; Simon et al., 1984; Wieloch, 1985; Weiss et al., 1986; Clark and Rothman, 1987; Gill et al., 1987), NMDA receptor blockers can prevent neuronal damage. Endogenous EAAs have been proposed as possible etiological agents in a variety of degenerative neuropathologies (Schwarcz et al., 1984; Ben-Ari, 1985; Beal et al., 1986; Maragos et al., 1987; Olney, 1987).

In view of the involvement of EAAs in long-lasting physiological modulation and in neuronal degeneration, the intraneuronal consequences of EAA activation are of primary interest. Studies of the mechanisms of synaptic potentiation and of neurotoxicity have pointed to EAA promotion of calcium influx as a potential triggering stimulus. Long-term potentiation cannot be induced in low calcium-containing media (Dunwiddie and Lynch, 1979) and is prevented by injections of the calcium chelator EGTA into the postsynaptic neuron (Lynch et al., 1983). Similarly, a number of in vitro studies have found that removal of extracellular calcium will block EAA neurotoxicity (Choi, 1985, 1987; Garthwaite et al., 1986; Rothman et al., 1987), although reports have also described for cultured neurons a rapid, chloride-dependent swelling and osmotic lysis that does not require calcium (Price et al., 1985; Rothman, 1985; Choi, 1987). The preferential involvement of the NMDA receptor subtype in these modifications also supports a role for calcium entry, as this receptor gates a channel capable of transporting calcium (MacDermott et al., 1986; Jahr and Stevens, 1987).

Because many enzymes and proteins are regulated by calcium, the number of processes activated upon EAA stimulation that potentially contribute to the neurotoxic mechanism is large. Recently, we obtained direct evidence in vivo for activation by EAAs of one such calcium-dependent process, stimulation of the calcium-activated neutral cysteine protease calpain I (Siman and Noszek, 1988). Calpain I activation produced increases of up to 8-fold in levels of proteolytic fragments of the substrate protein brain spectrin, which could be detected and quantified by immunoblot analysis using spectrin antibodies. A role for a degradative, irreversibly acting enzyme such as calpain I in neurodegenerative processes is an intuitively attractive possibility, and has received experimental support of 2 kinds. First, calpain activation appears to be a requisite for agonist-induced atrophy of another excitable tissue, skeletal muscle (Leonard and Salpeter, 1982). Second, neuronal populations in brain susceptible to degeneration contain relatively high concentrations of calpain I-like immunoreactivity (Siman et al., 1985a). In the 
present study, we have examined the relationship between activation of calpain I and production of hippocampal damage by the EAAs kainate and NMDA. We have compared the 2 phenomena with regard to their time courses, dose dependencies, sensitivities to antagonist, and localizations within hippocampal subfields, and we have tested the effect of a non-EAA hippocampal neurotoxin on calpain I activation.

\section{Materials and Methods}

\section{Materials}

Antibodies against the $\alpha$-subunit of rat brain spectrin (also known as fodrin) were prepared and affinity-purified from immune sera as previously described (Siman et al., 1985b, 1987; Siman and Noszek, 1988). CPP was obtained from Tocris (Essex, England). Electrophoresis chemicals and nitrocellulose were from BioRad (Richmond, CA). All other chemicals were purchased from Sigma (St. Louis, MO) or Fisher (Malvern, $\mathrm{PA})$.

\section{Methods}

Excitant administration. Adult male Sprague-Dawley rats (300-400 gm) were anesthetized with chloropent $(0.3 \mathrm{ml} / 100 \mathrm{gm})$ and received injections into the lateral ventricle of kainate or NMDA alone or in combination with CPP, or of colchicine, delivered through a $1 \mu$ l Hamilton syringe. Stereotaxic coordinates were $1.4 \mathrm{~mm}$ lateral to the bregma, anterior-posterior at the bregma, and $3.4 \mathrm{~mm}$ ventral to the surface of the brain. Successful intraventricular injections of the EAAs characteristically produced limbic seizures accompanied by vibrissae and upperbody tremor, eye distention, limb clonus, and occasional wet-dog shakes lasting up to $5 \mathrm{hr}$.

Histology. At $3,6,18$, or $24 \mathrm{hr}$ or 2,4 , or $5 \mathrm{~d}$ after excitatory amino acid administration, rats were deeply anesthetized with sodium pentobarbital and perfused intracardially with ice-cold $0.1 \mathrm{M}$ sodium phosphate, pH 7.4 (PB), followed by $4 \%$ paraformaldehyde in PB. Brains were postfixed for 4-7 d and were then cryoprotected overnight in $25 \%$ sucrose in PB. Coronal $35 \mu \mathrm{m}$ sections were prepared with a freezing microtome, taken from the injection track extending caudally through the entire dorsal hippocampus. Sections were either mounted and stained with cresyl violet or were stained free-floating by the silver-impregnation methods of Fink and Heimer (procedure II, 1967) or Nadler and Evenson (1983). Sections were mounted onto subbed slides, dehydrated, delipidated, and coverslipped, and they were examined and photographed with a Nikon Microphot light microscope and camera system.

Quantitation of proteolysis. Degradation of the calpain I substrate brain spectrin (fodrin) was determincd by a quantitative immunoblotting method as previously described (Siman and Noszek, 1988). Briefly, the septal third of each hippocampus was homogenized in $20 \mathrm{~mm}$ Tris$\mathrm{HCl}$ (pH 7.4) containing $1 \mathrm{~mm}$ EDTA, $1 \mathrm{~mm}$ EGTA, $0.1 \mathrm{~mm}$ phenylmethylsulfonylflouride, $0.1 \mathrm{~mm}$ leupeptin and centrifuged to generate crude membrane and cytosolic fractions. Cytosolic polypeptides were separated by SDS-PAGE and transferred to nitrocellulose paper by Western blotting. Proteolytic fragments of the $\alpha$-subunit of brain spectrin were detected on the paper by immunostaining using polyclonal antibodies against rat brain $\alpha$-spectrin and a streptavidin-biotin-horseradish peroxidase procedure. Breakdown of brain spectrin by calpain I produces 4 characteristic lower molecular-weight fragments $(M, \sim 140$ $150 \mathrm{kDa})$ that react with antibodies to the intact $\alpha$-subunit $\left(M_{r} \sim 240\right.$ $\mathrm{kDa}$ ). Levels of the 2 most prominent fragments (breakdown products 2 and 3; see Siman and Noszck, 1988) wcre quantified by scanning densitometry with an Ultroscan XL densitometer (LKB) connected to an IBM XT computer. GelScan software (LKB) was used to integrate the areas under peaks.

For localization of spectrin proteolysis in hippocampal subfields, the septal third of each hippocampus was placed into ice-cold $20 \mathrm{~mm}$ Tris$\mathrm{HCl}(\mathrm{pH} 7.4), 0.15 \mathrm{M} \mathrm{NaCl}, 2 \mathrm{~mm}$ EGTA, $50 \mu \mathrm{M}$ leupeptin, $0.1 \mathrm{~mm}$ phenylmethylsulfonylfluoride and cut into 1-2 mm transverse sections by hand. Each slice was laid flat and, under a dissecting microscope, 2 additional cuts were made, one horizontally and the other vertically at the junction of the hippocampal fissure and the lateral edges of the 2 blades of the dentate gyrus. Quadrants from each of the transverse sections were pooled and processed as described above for septal hippocampi.

\section{Results}

Selective antagonism of NMDA-induced hippocampal damage and calpain I activation

Intracerebroventricular injections of kainate or NMDA induce seizure activity, hippocampal damage (Nadler et al., 1978, 1980, 1981) and degradation of the protein brain spectrin through activation of the calcium-dependent cystcine protease calpain I (Siman and Noszek, 1988). First, we examined if these effects of kainate or NMDA could be pharmacologically distinguished. Five days after i.c.v. administration of either kainate $(1 \mu \mathrm{g}$; Fig. $1 A)$ or NMDA ( $80 \mu \mathrm{g}$; Fig. $1 C)$ alone, large numbers of pyramidal neurons in area CA3 within the septal half of ipsilateral hippocampus had degenerated and been cleared, confirming previous studies (Nadler et al., 1978, 1980, 1981). Because of its selective and potent antagonism of NMDA receptors (Davies et al., 1986; Harris et al., 1986), carboxypiperazin-4-yl-propylphosphonate (CPP) was coadministered to rats along with NMDA or kainate. CPP at $2 \mu \mathrm{g}$ completely prevented the neuronal loss induced by NMDA in 3 of 3 cases (Fig. $1 D$ ) but had no discernible effect on the neurotoxicity of kainate in 3 of 3 instances (Fig. $1 B$ ). This suggests that NMDA exerts its neurotoxic effect by binding to a receptor class distinct from that activated by kainate.

The effects of CPP on kainate- or NMDA-induced calpain I activation were determined by quantitation of the levels of major proteolytic fragments of the calpain I substrate brain spectrin. The $\alpha$-subunit of brain spectrin and its smaller proteolytic breakdown products were detected by an immunoblot procedure. As shown in Figure 2, hippocampus of kainate- or NMDAtrealed rats contained, in addition to the $M_{r} \sim 240 \mathrm{kDa} \alpha$-subunit of brain spectrin, several prominent immunoreactive $\alpha$-spectrin breakdown products (BDPs) of $M_{i} \sim 140-150 \mathrm{kDa}$. This specific pattern of BDPs results from spectrin degradation by the calcium-dependent cysteine protease calpain I (Siman and Noszek, 1988). Spectrin proteolysis could be quantified as the increase in BDP levels measured by scanning densitometry, a method that responds linearly to increases in BDP content over a 10 fold range (Fig. $2 A$ ). Twenty-four hours after i.c.v. injections of kainate $(1 \mu \mathrm{g})$ or NMDA $(80 \mu \mathrm{g})$, BDP levels increased more than 7-fold (Fig. 2, B, C). Coadministration of CPP at $2 \mu \mathrm{g}$ completely blocked NMDA-induced spectrin breakdown. In contrast, at 2 or even $5 \mu \mathrm{g}$ doses, CPP had little effect on the calpain I activation induced by kainate. Thus, as was the case with its effects on neuronal death, CPP selectively antagonized NMDA- but not kainate-induced spectrin proteolysis.

While $2 \mu \mathrm{g}$ CPP completely inhibited the neuronal damage and calpain I activation induced by $80 \mu \mathrm{g}$ NMDA, the behavioral manifestations of limbic seizure activity, such as forelimb clonus, eye distention, and vibrissae and upper-body tremor, were not substantially affected. This suggests that calpain I activation is more closely associated with hippocampal damage than with seizure activity per se (see also below).

\section{Dose-responses for kainate- and NMDA-induced neurotoxicity and calpain I activation}

In further examining the relationship between protease activation and the production of neuronal damage, we determined the capacities of various doses of kainate or NMDA to evoke the 2 phenomena. Injection of $0.25 \mu \mathrm{g}$ kainate produced a readily detectable loss of CA3 pyramidal neurons within $5 \mathrm{~d}$ in Nisslstained material (Fig. 3), particularly in the septal third of hip- 

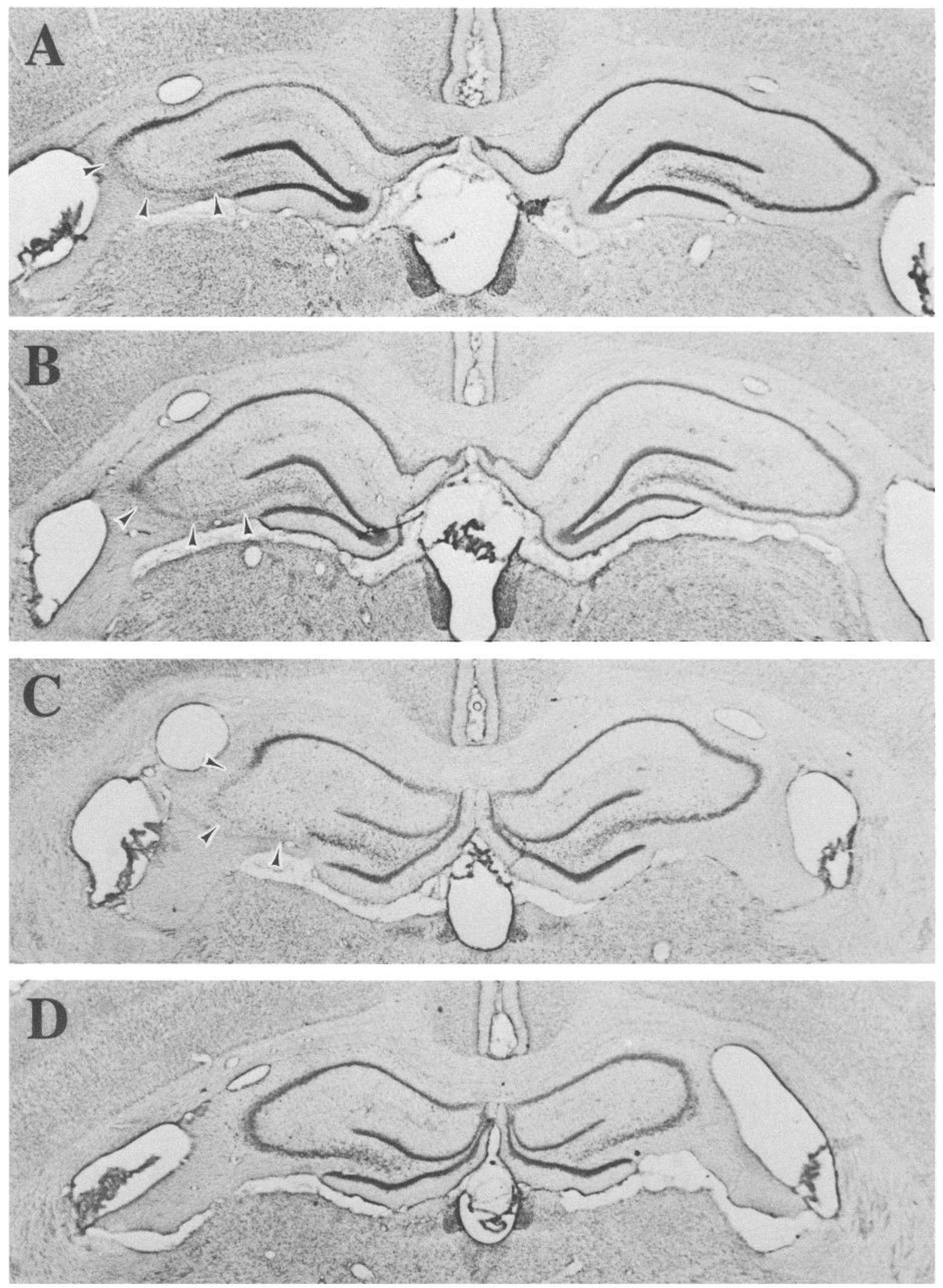

Figure 1. Selective antagonism of NMDA- but not kainate-induced neurotoxicity by CPP. Nissl-stained coronal sections taken through dorsal hippocampus of rats injected intraventricularly $5 \mathrm{~d}$ earlier with $(A) 1 \mu \mathrm{g}$ kainate, $(B) 1 \mu \mathrm{g}$ kainate $+2 \mu \mathrm{g}$ CPP, $(C) 80 \mu \mathrm{g}$ NMDA, or $(D) 80 \mu \mathrm{g}$ NMDA $+2 \mu \mathrm{g}$ CPP. In each case, hippocampus ipsilateral to the injection is shown at left. Arrowheads denote zones of extensive pyramidal neuronal loss in area CA3. Note that CPP prevented the NMDA-induced neurodegeneration but had little effect on the neurotoxicity of kainate. This experiment was replicated twice. $\times 10$. 
Figure 2. Quantitation of spectrin proteolysis and selectivity of its antagonism by CPP. $A$, Immunoblot of polypeptides from ipsilateral hippocampus $24 \mathrm{hr}$ after i.c.v. injection of $1 \mu \mathrm{g}$ kainate, stained with antibodies to rat brain $\alpha$-spectrin. Shown here are the major $\alpha$-spectrin proteolytic fragments $\left(M_{r} \sim 140-150 \mathrm{kDa}\right)$ resulting from cleavage by calpain I. Each lane was loaded with the indicated volume $(\mu \mathrm{l})$ of hippocampal homogenate. The detection of spectrin breakdown product levels is linear over about a 10 -fold concentration range. $B$, Representative immunoblots illustrating that NMDA markedly stimulates hippocampal spectrin degradation, and this effect is blocked by co-injection of CPP. Each lane is prepared from a different rat. $C$, Summary of results obtained from 3-8 rats (means \pm SEM) per experimental condition. CPP completely and selectively prevented NMDA- but not kainate-stimulated spectrin degradation. The NMDA + CPP condition is significantly different from both of the kainate + CPP conditions $(p<0.01)$.

\section{A}
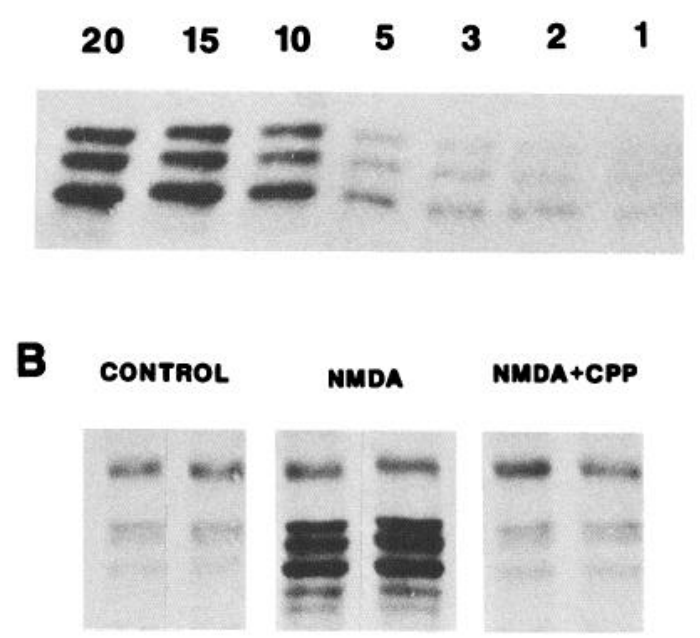

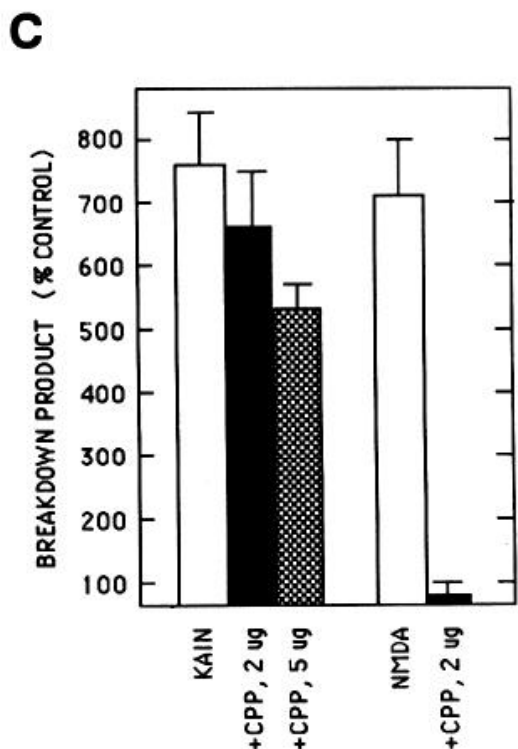

pocampus. Kainate-induced damage became more severe at 0.5 and $1 \mu \mathrm{g}$ doses, which destroyed more dentate hilar and area CA1 neurons in the septal third (Fig. 3), as well as more area CA3 pyramids in the midseptotemporal third. This is fully consistent with the previous extensive studies of Nadler and colleagues $(1978,1980,1981)$ on the toxicity of intraventricular kainate.

Compared with kainate, NMDA was a substantially less potent hippocampal neurotoxin (Fig. 4). At $24 \mathrm{hr}$ following injection of $20 \mu \mathrm{g}$ NMDA, only a few degenerating neurons could be detected with silver-impregnation histochemistry. This dose damaged a minority of neurons in area CA3 and in the infrapyramidal blade of the dentate gyrus, in close proximity to the lateral ventricle (Fig. 4, $A, C$ ). At 5 d postinjection, little ncuronal loss could be discerned in Nissl-stained sections, except for at the extreme septal pole. However, NMDA at $80 \mu \mathrm{g}$ caused massive hippocampal damage, destroying large numbers of infrapyramidal dentate granule and $\mathrm{CA} 3$ pyramidal cells throughout the septal third (Fig. 4, $B, D$ ), as well as extensive loss of CA1 neurons near the septal pole.

Those doses of kainate or NMDA found to produce extensive neuronal death also markedly stimulated calpain I-mediated spectrin degradation (Table 1). A small increase in spectrin breakdown product levels was apparent $24 \mathrm{hr}$ after $0.15 \mu \mathrm{g}$ kainate, but the maximal increase of more than 7 -fold required $1 \mu \mathrm{g}$ kainate (Table 1 ). Similarly, the relatively nonneurotoxic doses of NMDA, those below $80 \mu \mathrm{g}$, produced only small increases in spectrin breakdown, while the enormously neurotoxic does of $80 \mu \mathrm{g}$ elevated BDP levels by more than 7 -fold (Table 1). Among the 13 rats administered 20 or $40 \mu \mathrm{g}$ NMDA, only 3 showed an increase in spectrin BDP content of more than $100 \%$, as compared with 7 of 8 rats given $80 \mu \mathrm{g}$.

Intraventricular NMDA evoked seizure activity at the 20 and $40 \mu \mathrm{g}$ doses that caused little hippocampal damage. A similar discrepancy between the neurotoxic and convulsant properties of NMDA has previously been reported (Zaczek et al., 1981;
Zaczek and Coyle, 1982). Based on the failure of convulsant doses of NMDA to induce marked proteolysis, the induction of spectrin degradation corresponded more closely with the production of damage than of seizure activity. Additionally, as mentioned above, the NMDA antagonist CPP at $2 \mu \mathrm{g}$ blocked the neuronal damage and proteolysis without appreciably modifying the convulsions induced by NMDA. Thus, seizure activity alone is not sufficient to activate calpain I and induce spectrin degradation; protein breakdown occurs only under conditions that also destroy hippocampal neurons.

\section{Localization of calpain I-mediated spectrin proteolysis}

If the EAA induction of spectrin degradation is related to its production of hippocampal damage, then activation of spectrin proteolysis should be restricted to those areas in which neuronal damage is prominent. To examine this issue, we dissected hippocampus from control (uninjected) and kainate-treated rats into 4 quadrants, as shown in Figure $5 \mathrm{~A}$. Quadrant 3, which contains most of area CA3, exhibited the majority of neuronal degeneration. This region also showed, within $24 \mathrm{hr}$, the largest kainate-induced stimulation of spectrin degradation (Fig. 5, $B$, $C)$, with BDP levels increasing more than 7 -fold compared with quadrant 3 from untreated rats. Stimulation of proteolysis in quadrant 3 was more than 3 times that of quadrants 1 or 4 . In 5 of 5 experiments the largest increase in spectrin BDPs occurred in quadrant 3.

Two brain regions exhibiting little or no neuronal loss following i.c.v. kainate treatment, the striatum and prefrontal cortex (Nadler et al., 1978, 1980) were also examined for spectrin degradation. In these 2 areas there was no change in BDP levels (data not shown).

\section{Time courses of kainate-induced hippocampal damage and calpain I activation}

Calpain I activation could occur as an early, primary response to sustained EAA exposure or, alternatively, could merely be a 


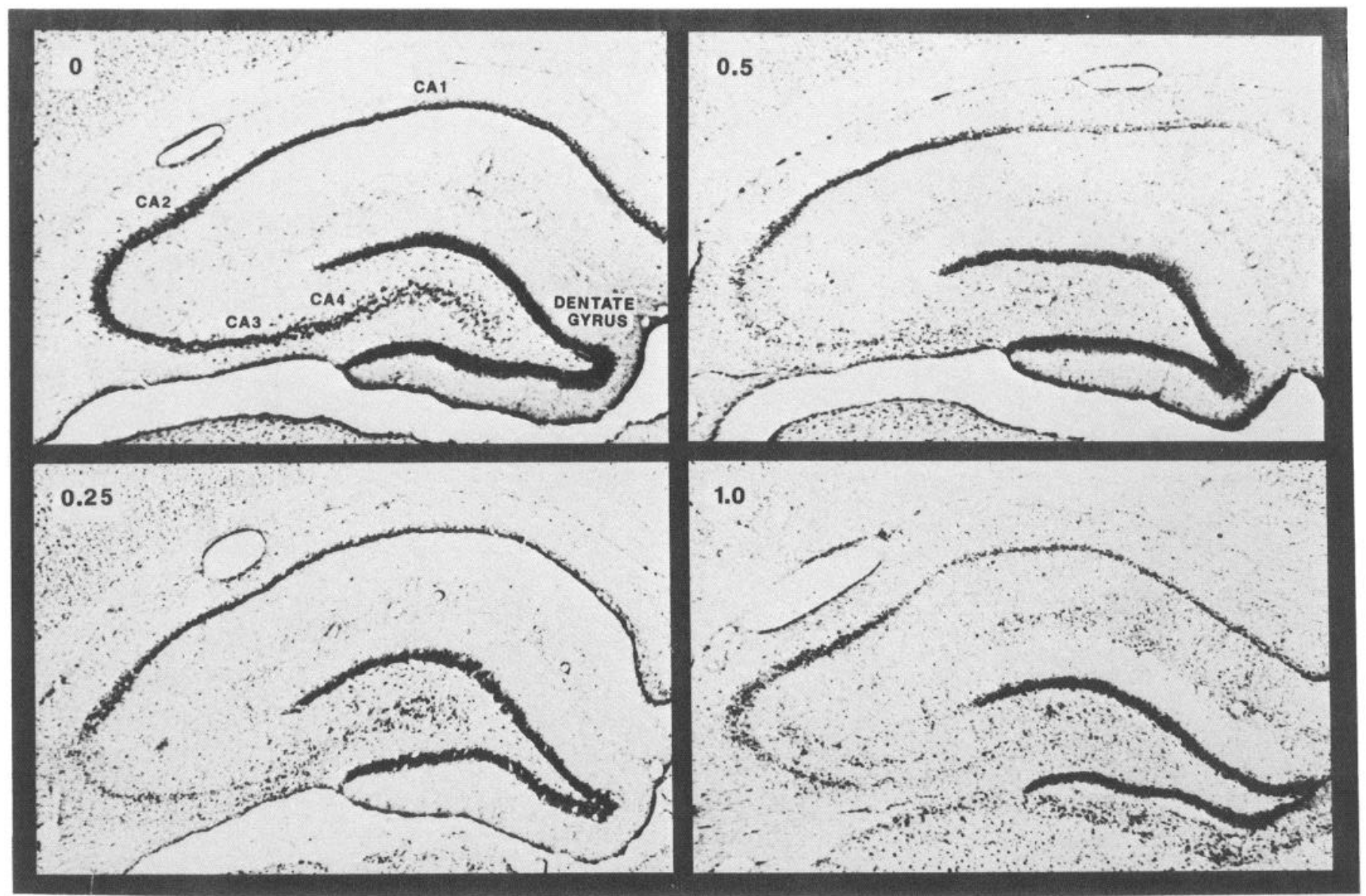

Figure 3. Dose dependence of kainate induction of hippocampal damage. Nissl-stained coronal sections through dorsal hippocampus taken $5 \mathrm{~d}$ after i.c.v. injection of the indicated doses of kainate (in $\mu \mathrm{g}$ ). Hippocampal subfields are indicated in the control $(O)$. Most pyramidal neurons in ipsilateral hippocampus in areas CA 3 and CA4 were destroyed by doses as low as $0.25 \mu \mathrm{g}$, while at doses of 0.5 and $1 \mu \mathrm{g}$ increasing numbers of dentate hilar and CA1 pyramidal neurons were affected. Pyramidal neurons in area CA2 and dentate granule cells were resistant to kainate neurotoxicity. $\times 10$.

secondary response initiated by the breakdown of neuronal integrity. As a means of distinguishing between these possibilities, we compared the temporal onset of spectrin proteolysis with that of neuronal degeneration. Rats were killed 3, 6, 18, 24, 48, or $72 \mathrm{hr}$ after receiving $1 \mu \mathrm{g}$ kainate i.c.v. and were used for either quantitation of spectrin breakdown or silver-impregnation histochemistry. As illustrated in Figure 6, at $3 \mathrm{hr}$ no degenerating argyrophilic profiles were observed at any septotemporal level. At $6 \mathrm{hr}$, only small numbers of pyramidal neurons, particularly those located laterally (stratum oriens of area CA3a) and a few nearest the dentate hilus, had begun to degenerate. In sharp contrast, by $18 \mathrm{hr}$ most CA3 perikarya and dendritic processes within the septal third were argyrophilic. Thus, according to the criterion of argyrophilia, neuronal degeneration became prominent between $6-18 \mathrm{hr}$ after kainate administration.

Stimulation of calpain I-induced spectrin degradation by kainate preceded the onset of argyrophilic neuronal degeneration. Spectrin BDP content had increased significantly within 3 $\mathrm{hr}$, and by $6 \mathrm{hr}$ the increase was half-maximal (Fig. 7). BDP levels peaked at $24 \mathrm{hr}$, remained near-maximal over the next 2 d (Fig. 7), and were still elevated at $9 \mathrm{~d}$, the longest time point examined (data not shown).

\section{Table 1. Dose dependence of excitatory amino acid-induced spectrin degradation}

\begin{tabular}{cl}
$\begin{array}{l}\text { Treatment } \\
(\mu \mathrm{g})\end{array}$ & $\begin{array}{l}\text { Spectrin BDP } \\
(\% \text { control } \pm \text { SEM })\end{array}$ \\
\hline Kainate & \\
0.15 & $255 \pm 34$ \\
0.25 & $420 \pm 180$ \\
0.5 & $657 \pm 213$ \\
1.0 & $765 \pm 70$ \\
NMDA & \\
5 & $126 \pm 23$ \\
20 & $343 \pm 130$ \\
40 & $318 \pm 170$ \\
80 & $709 \pm 103$ \\
\hline
\end{tabular}

Spectrin BDP levels were determined by quantititive immunoblot analysis of hippocampal cytosolic fractions from rats injected $24 \mathrm{hr}$ earlier with the indicated doses of EAAs; levels are compared to BDP levels from control, uninjected rats. Each value represents the mean of 4-8 determinations. Note that both kainate and NMDA induced dose-dependent increases in spectrin degradation, that kainate was substantially more potent than NMDA, and that the dose-responses for activation of proteolysis are similar to those for production of hippocampal neurotoxicity (Figs. 3, 4). 

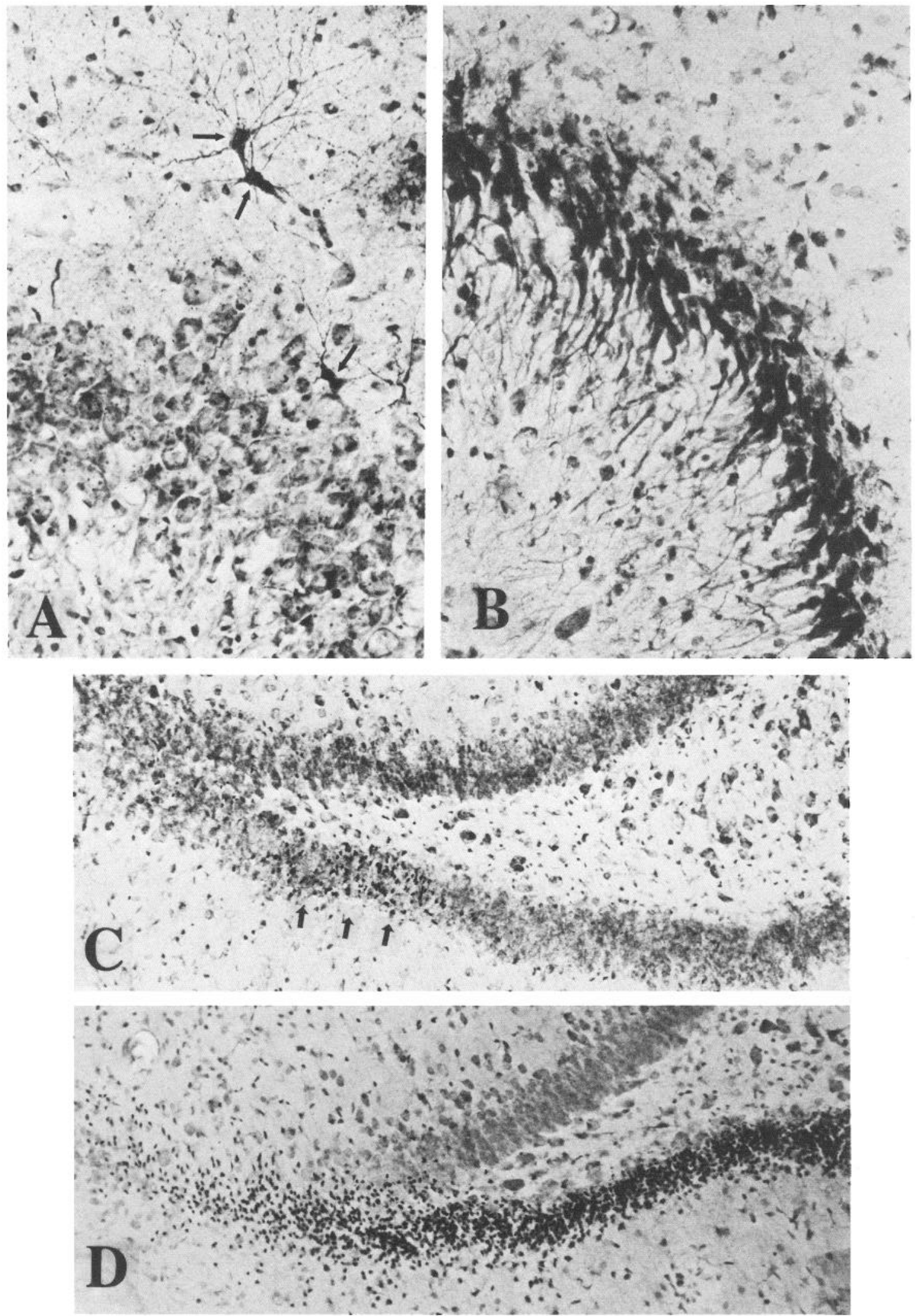

Figure 4. Dose dependence of NMDA induction of neuronal degeneration. Silver-impregnated sections taken $24 \mathrm{hr}$ after administration of $(A, C)$ $20 \mu \mathrm{g}$ or $(B, D) 80 \mu \mathrm{g}$ NMDA. $A$ and $B$, area CA3; $C$ and $D$, dentate gyrus. At $20 \mu \mathrm{g}$, only a few neurons in stratum oriens of area CA3 close to the lateral ventricle and in the dentate granule cell layer were argyrophilic (arrows). In contrast, $80 \mu \mathrm{g}$ NMDA caused massive degeneration in area CA3 $(B)$ and the infrapyramidal blade of the dentate gyrus $(D)$. Magnification: $A$ and $B, \times 200 ; C$ and $D, \times 50$. 


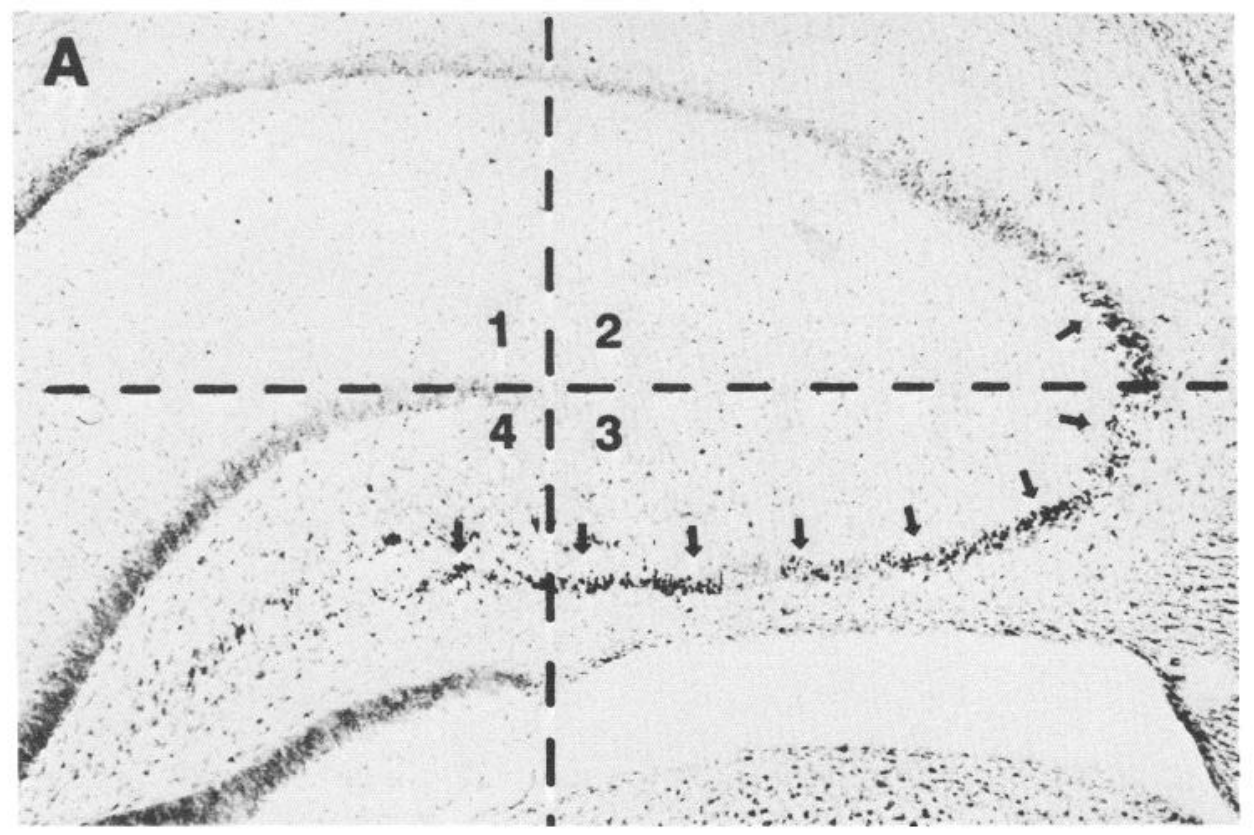

B

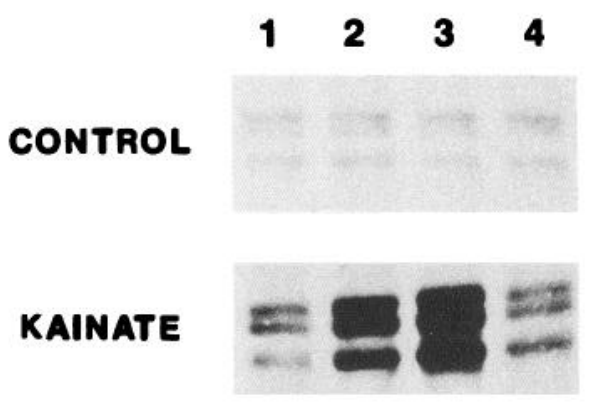

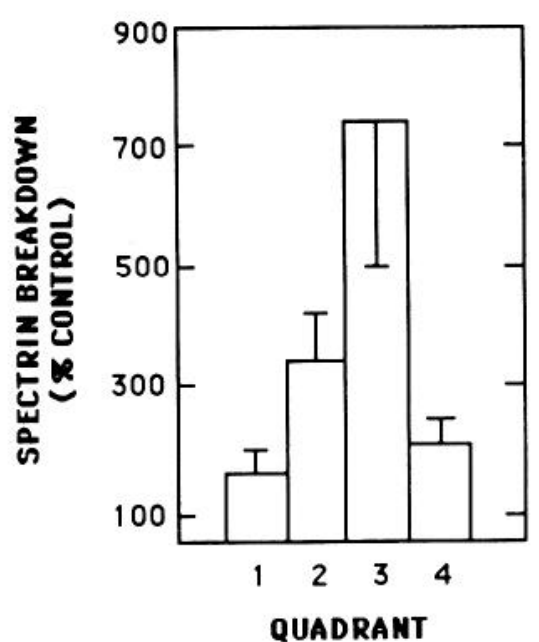

Figure 5. Localization of calpain I-mediated spectrin degradation in hippocampal subfields. A, Silver impregnation illustrating positions of horizontal and vertical cuts made to microdissect hippocampus into 4 quadrants, and of extent of neuronal damage in each quadrant. Argyrophilic neurons are primarily in area CA3 (arrows) in quadrant 3. $B$, Detection of spectrin degradation in each quadrant by immunoblot analysis. Top panel shows the 4 quadrants from a control, untreated rat; bottom panel, results from a rat administered $1 \mu \mathrm{g}$ kainate $24 \mathrm{hr}$ earlier. $C$, Summary of spectrin degradation results from 5 experiments. Each value represents the mean \pm SEM. In all 5 cases, the largest spectrin breakdown occurred in quadrant 3.

\section{Colchicine causes hippocampal damage without activating calpain I}

For further testing of the specificity with which EAAs induce calpain I-mediated spectrin degradation, we determined whether hippocampal damage produced by a non-EAA, colchicine (Goldschmidt and Steward, 1980), would be accompanied by spectrin breakdown. Injection of $20 \mu \mathrm{g}$ colchicine i.c.v. caused massive degeneration of granule neurons in ipsilateral dentate gyrus. At $48 \mathrm{hr}$ postinjection, many of these neurons were argyrophilic (Fig. $8 \mathrm{~A}$ ), and by $4 \mathrm{~d}$, Nissl staining revealed clearance of large numbers of cells. In addition, variable numbers of CA1 and CA3 pyramidal neurons were affected, especially those situated nearest the lateral ventricle. Despite the considerable neuronal damage, spectrin BDP levels increased by only 7 and $43 \%$ by 24 and $48 \mathrm{hr}$, respectively, compared with the more than
$600 \%$ increase induced by NMDA (Fig. $8 B$ ). Thus, calpain I-mediated spectrin degradation does not occur under all conditions leading to hippocampal neurotoxicity.

\section{Discussion}

Here we have evaluated the relationship between the capacities of the excitatory amino acids kainate and NMDA to induce hippocampal damage and to activate calcium-dependent proteolysis of the protein brain spectrin. We have found that, under a variety of conditions, there is a close correspondence between the induction of the 2 phenomena by EAAs (Table 2). The correspondences include sensitivities to a receptor antagonist, dose-responses, time courses, and localizations within hippocampal subfields. The results support our earlier proposal (Siman and Noszek, 1988) that excitatory neurotransmission may regulate intraneuronal protein catabolism. Further, they suggest 

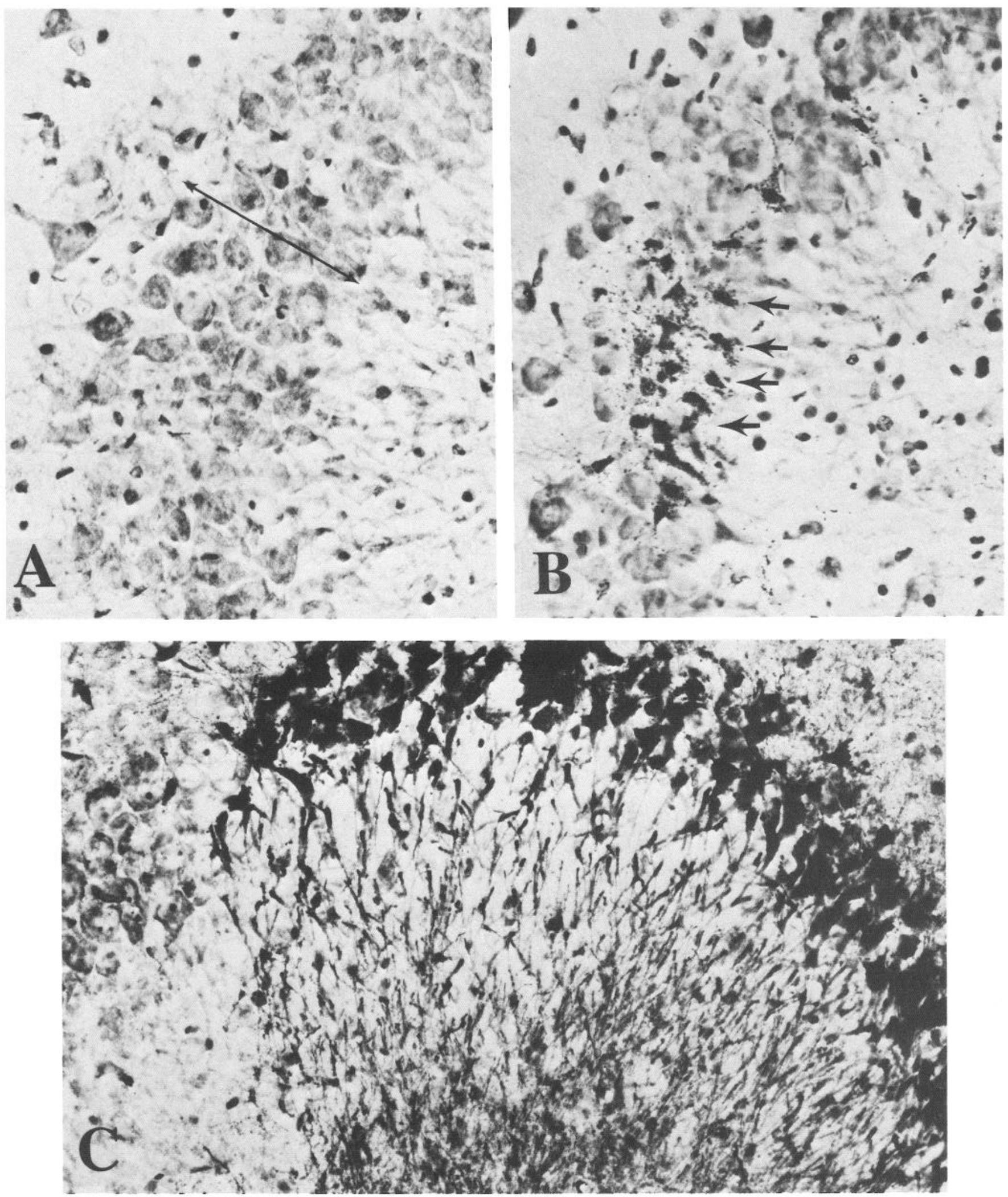

Figure 6. Time course of kainate-induced neuronal degeneration. Area CA3 is shown from silver-stained sections taken from ipsilateral hippocampus of rats treated with $1 \mu \mathrm{g}$ kainate for $(A) 3 \mathrm{hr},(B) 6 \mathrm{hr}$, or $(C) 18 \mathrm{hr}$. Arrow in $A$ denotes pyramidal cell layer. Note that by $6 \mathrm{hr}$, only a minority of neurons were argyrophilic (arrows), while essentially all neurons of area CA3 were degenerating by $18 \mathrm{hr}$. $\times 200$. 
Table 2. Correspondence between induction of neuronal damage and of calpain I-mediated spectrin degradation

\begin{tabular}{lll} 
Condition & Neurotoxicity & $\begin{array}{l}\text { Calpain } \\
\text { activation }\end{array}$ \\
\hline Kainate, $0.25 \mu \mathrm{g}$ & + & + \\
Kainate, $1 \mu \mathrm{g}$ & ++ & ++ \\
$\mathrm{NMDA}, 40 \mu \mathrm{g}$ & + & + \\
$\mathrm{NMDA}, 80 \mu \mathrm{g}$ & ++ & ++ \\
Kainate, $1 \mu \mathrm{g},+\mathrm{CPP}, 2 \mu \mathrm{g}$ & ++ & ++ \\
NMDA, $80 \mu \mathrm{g},+\mathrm{CPP}, 2 \mu \mathrm{g}$ & - & - \\
Kainate, $1 \mu \mathrm{g}(6 \mathrm{hr})$ & + & + \\
Kainate, $1 \mu \mathrm{g}$ & Area CA3 & Area CA3 \\
Colchicine, $20 \mu \mathrm{g}$ & ++ & - \\
\hline
\end{tabular}

Summary of results illustrating that the EAA-induced activation of calpain I is closely correlated with EAA production of hippocampal neuronal damage. Only the non-EAA colchicine evokes a degree of neurotoxicity that is not matched by the degree of proteolysis. ++ , maximal effect; + , modest effect; - , little or no effect.

that calcium-dependent proteolysis serves as an intracellular mediator of excitatory amino acid action.

Studies in tissue culture have defined 2 distinct mechanisms by which EAAs may be neurotoxic. One is activated by 30 or more minutes of continuous EAA exposure, develops rapidly, and is dependent on extracellular chloride (Price et al., 1985; Rothman, 1985; Choi, 1987). The second is activated by brief (5-10 min) EAA treatment, develops slowly over many hours, and requires extracellular calcium (Choi, 1985, 1987; Rothman et al., 1987). The present results indicating that spectrin degradation is stimulated by neurotoxic EAA treatment, coupled with the previous demonstration that this degradation is mediated by the calcium-dependent protease calpain I (Siman and Noszek, 1988), provide evidence that the in vitro calcium-dependent mechanism more closely models EAA neurotoxicity in vivo and suggest that the intraneuronal process activated by calcium to trigger the neuronal damage may be proteolysis.

Strong evidence associating calpain I activation with EAA neurotoxicity is provided by the dose dependencies for the 2 processes. Only those doses of kainate $(0.5-1 \mu \mathrm{g})$ or NMDA ( 80 $\mu \mathrm{g}$ ) that, within 4-5 d, produced extensive pyramidal cell loss also markedly activated calpain I-mediated spectrin degradation. Lower doses of kainate induced graded, decreasing amounts of damage and proteolysis. Both responses to NMDA declined sharply below $80 \mu \mathrm{g}$, and either a low dose of NMDA $(20 \mu \mathrm{g})$ or a high dose $(80 \mu \mathrm{g})$ paired with a low dose of CPP $(2 \mu \mathrm{g})$ caused seizures without inducing substantial neuronal damage or calpain I activation. In addition, we have recently found that convulsant but nontoxic doses of the GABA antagonist bicuculline do not cause calpain I activation (data not shown). Our observations confirm previous studies using other sites of EAA administration (Zaczek et al., 1981; French et al., 1982) and convulsant drugs (Gruenthal et al., 1986) which indicate that seizure activity per se is insufficient to produce neuronal damage. Similarly, calpain I activation is not induced merely in response to seizures, but its expression requires conditions that lead to neuronal loss.

\section{$\begin{array}{llllllll}0 & 0 & 3 & 6 & 18 & 24 & 48 & 72\end{array}$}
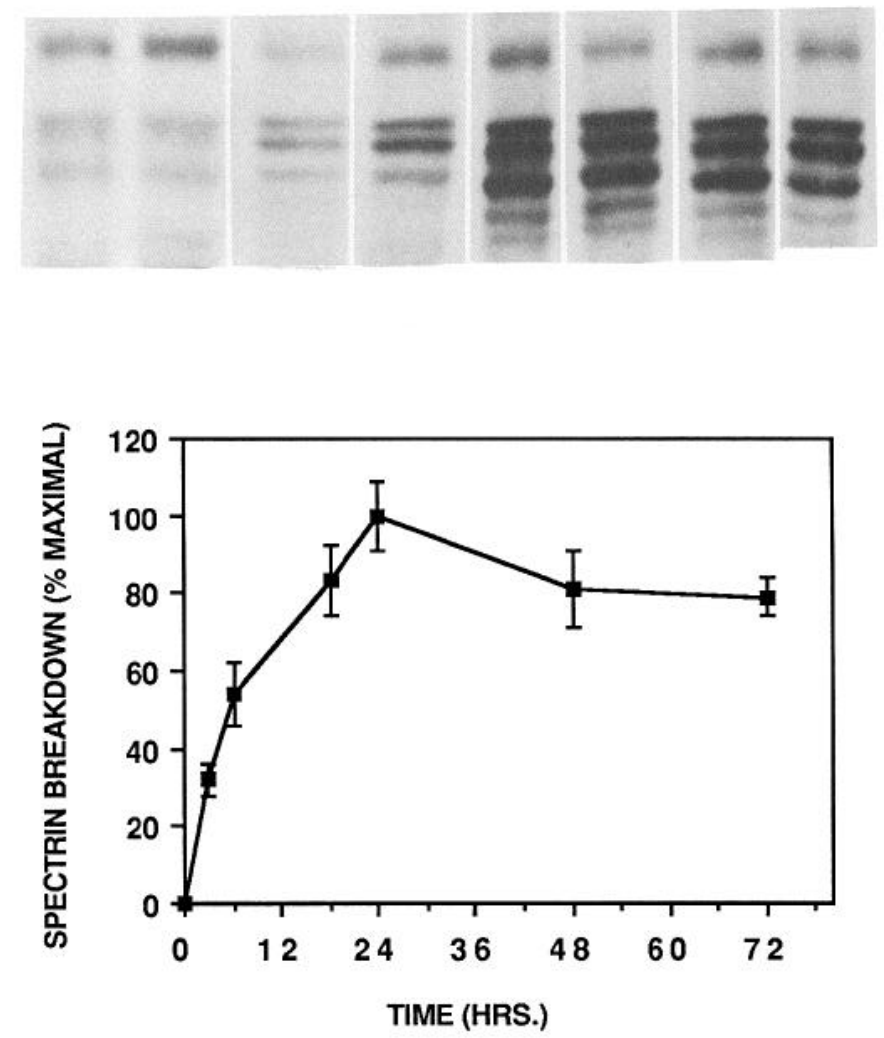

Figure 7. Time course of kainate-induced calpain I activation. Top, Representative immunoblot showing content of spectrin breakdown products at the indicated times (in hr) after i.c.v. injection of $1 \mu \mathrm{g}$ kainate. Bottom, Summary of results from 3 experiments, which differed from one another by less than $20 \%$. Each value is the mean \pm SEM. Spectrin breakdown was approximately half-maximal by $6 \mathrm{hr}$, peaked at $24 \mathrm{hr}$, and remained elevated thereafter.

The time course and localization of protease activation also suggest a direct relationship to neuronal degeneration. Protease activation is localized primarily to the CA3 region of hippocampus that exhibits the greatest susceptibility to neuronal damage in response to i.c.v. EAAs (Fig. 5). Within this area, spectrin degradation is elevated by $3 \mathrm{hr}$ and is half-maximal at $6 \mathrm{hr}$ (Fig. 7 ), times at which few degenerating neurons can be detected by silver-impregnation histochemistry (Fig. 6). A similar rapid protease activation has been observed following systemic kainate administration (Siman and Noszek, 1988). By the criterion of argyrophilia, then, calpain I activation precedes the neurodegeneration. It should be noted, however, that ultrastructural studies detect subtle changes as early as $30 \mathrm{~min}$ after EAA treatment, in the form of dilation of endoplasmic reticulum coupled with dispersion of polyribosomes (Hattori and McGeer, 1977; Olney et al., 1979; Herndon et al., 1980; Nadler et al., 1980; Hajos et al., 1986). Over the next few hours, structural damage progresses to include clumping of nuclear chromatin, swelling of dendrites, their spines, and astrocytic processes, and pyknosis of neuronal perikarya. The precise role that protease activation or other molecular events may play in these initial ultrastructural changes has yet to be defined.

While the present results clearly demonstrate a relationship between the EAA induction of neuronal damage and of calpain 
Figure 8. Colchicine lesions are not accompanied by calpain I activation. $A$, Extensive granule cell degeneration evoked $48 \mathrm{hr}$ after i.c.v. administration of $20 \mu \mathrm{g}$ colchicine. Neurons in both the suprapyramidal (arrows) and infrapyramidal blade had begun to degenerate, while dentate hilar neurons $(d h)$ were spared. $\times 40 . B$, Immunoblot of hippocampal polypeptides taken from uninjected rats (lanes $a, b$ ) or rats exposed for $24 \mathrm{hr}$ to colchicine, $20 \mu \mathrm{g}$ (lanes $c, d$ ), or NMDA, $80 \mu \mathrm{g}$ (lane $e$ ).
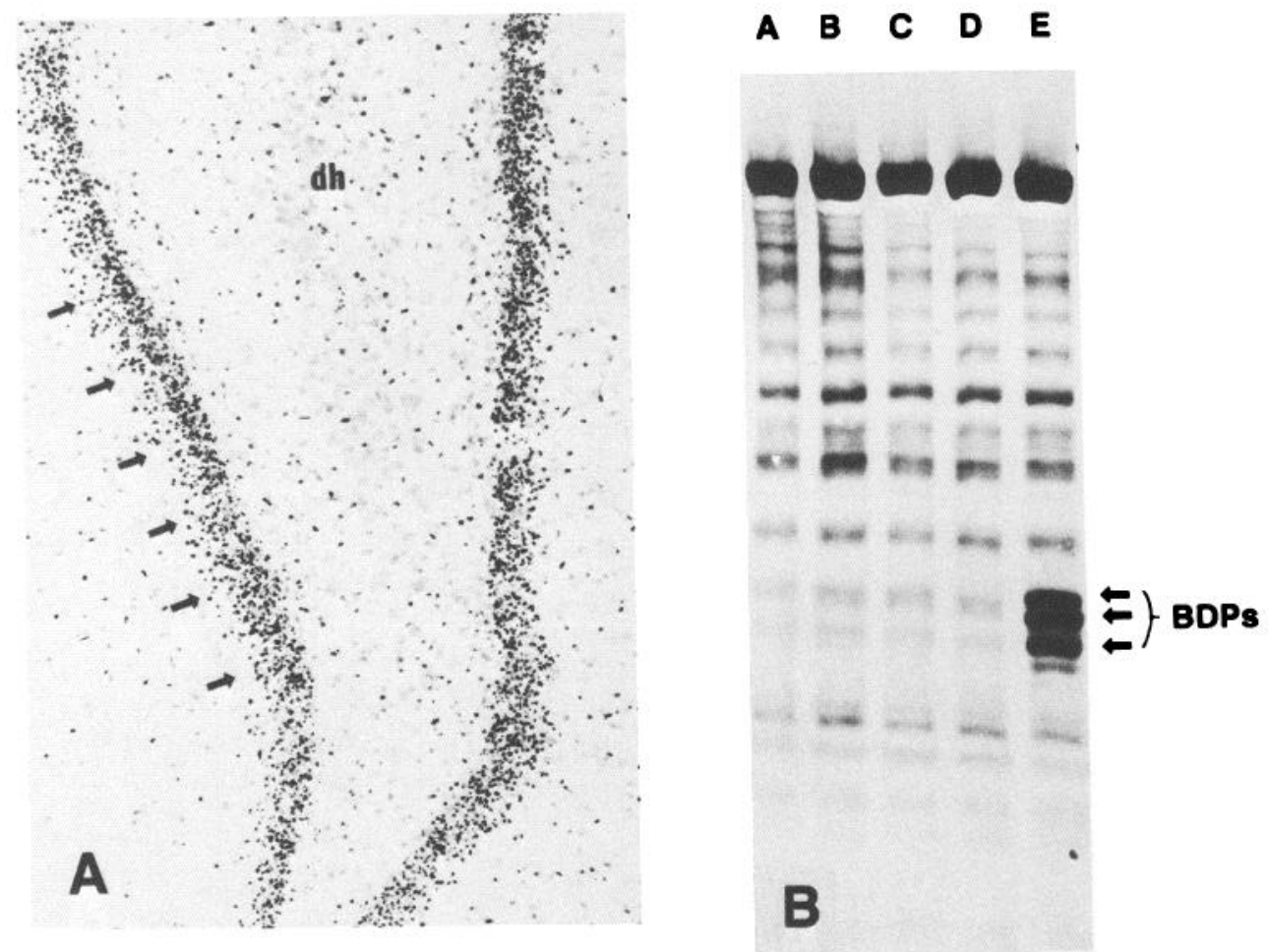

I activation, the exact nature of this relationship is not established. Three possibilities exist. First, calcium-activated proteolysis may simply occur in response to structural disintegration of neurons. Second, proteolysis could be a direct, primary response to EAA stimulation but may not be directly involved in the neurotoxic process. Third, calpain I activation may be a primary and obligatory event in the neurodegenerative mechanism. The first possibility is unlikely in view of the finding that hippocampal neuronal damage caused by the non-EAA colchicine is not accompanied by calpain I activation (Fig. 8). The rapid onset of protease activation also argues against this possibility. It is also unlikely that protease activation is a primary response to EAA exposure but is not involved in the neurodegeneration. Only those doses of EAAs that cause hippocampal damage also activate calpain I, with neuronal excitation and seizure activity alone being insufficient to evoke the 2 phenomena. Therefore, the most likely possibility is that protease activation is an early, requisite step in the intracellular cascade by which EAAs induce neuronal death. Experiments designed to inhibit EAA-stimulated proteolysis or to activate calpain I independent of EAAs could be used to test this hypothesis critically.

The steps subsequent to calpain I activation that may contribute to the neurodegenerative process remain to be identified. Our studies have focused on degradation of the protein brain spectrin (also called fodrin), a major structural component of neurons (reviewed by Bennett, 1985; Goodman and Zagon, 1986). Spectrin is thought to be a primary component of a submembraneous protein skeleton that shares structural and functional homology with the erythrocyte cytoskeleton, and which may control nonerythroid cell shape and membrane protein and lipid organization (Levine and Willard, 1983; Bourguignon et al., 1985; Siman et al., 1985b; DelBuono et al., 1988; Srinivasan et al., 1988). Conceivably, then, spectrin degradation could bring about localized collapse of the protein skeleton and overlying membrane, thereby initiating neuronal structural disintegration. However, in vitro studies indicate that calpain I can use as substrates large numbers of proteins of structural and metabolic importance (Zimmerman and Schlaepfer, 1984; Siman and Noszek, 1988), and the breakdown of any or several of these may participate in the death of a neuron. In this regard, it is important to note that not all in vitro calpain I substrates are capable of being degraded upon calpain I activation in vivo, as proteolysis is constrained by the restricted localizations of the calcium currents, the protease and its preferred substrates (Siman and Noszek, 1988). Our present knowledge in these areas is inadequate to predict the full complement of calpain substrates that may be degraded in response to EAA activation in vivo.

In addition to its possible role in pathological neurodegeneration, EAA-induced calpain I activation may be of physiological importance. Two forms of plasticity in adulthood require NMDA receptor activation, long-term potentiation of synaptic transmission (Collingridge et al., 1983; Harris et al., 1984; Artola and Singer, 1987), and visual cortical ocular dominance (Kleinschmidt et al., 1987). These NMDA-mediated modifications may involve synaptic structural changes (Lynch and Baudry, 1984). EAAs also modulate the structure of dendritic processes of developing neurons by a calcium influx-dependent mechanism (Mattson et al., 1988). The robust EAA-induced calpain activation that may participate in neurodegenerative structural alterations could, when stimulated to a more modest degree, produce these subtle morphological changes. Although, in the present study, doses of EAAs that cause hippocampal damage were required for markedly promoting calpain I-induced spectrin degradation, the detection of more restricted proteolysis occurring in response to moderate physiological stimuli may require an anatomical or biochemical method ca- 
pable of localizing proteolysis within distinct subcellular compartments.

\section{References}

Artola, A., and W. Singer (1987) Long-term potentiation and NMDA receptors in rat visual cortex. Nature 330: 649-652.

Beal, M. F., N. W. Kowall, D. W. Ellison, M. F. Mazurek, K. J. Swartz, and J. B. Martin (1986) Replication of the neurochemical characteristics of Huntington's disease by quinolinic acid. Nature 321: 168171.

Ben-Ari, Y. (1985) Limbic seizure and brain damage produced by kainic acid: Mechanisms and relevance to human temporal lobe epilepsy. Neuroscience 14: 375-403.

Bennett, V. (1985) The membrane skeleton of human erythrocytes and its implications for more complex cells. Annu. Rev. Biochem. 54: 273-304.

Bourguignon, L. Y. W., S. J. Suchard, M. L. Magpal, and J. R. Glenney, Jr. (1985) A T-lymphoma transmembrane glycoprotein (gp 180) is linked to the cytoskeletal protein, fodrin. J. Cell Biol. 101: 477-487.

Choi, D. W. (1985) Glutamate neurotoxicity in cortical cell cultures is calcium-dependent. Neurosci. Lett. 58: 293-297.

Choi, D. W. (1987) Ionic dependence of glutamate neurotoxicity. J. Neurosci. 7: 369-379.

Clark, G. D., and S. M. Rothman (1987) Blockade of excitatory amino acid receptors protects anoxic hippocampal slices. Neuroscience 21 : 665-671.

Collingridge, G. L., S. J. Kehl, and H. McLennan (1983) Excitatory amino acids in synaptic transmission in the Schaffer collateral-commissural pathway of the rat hippocampus. J. Physiol. (Lond.) 334: 33-46.

Davies, J., R. H. Evans, P. L. Herrling, A. W. Jones, H. J. Olverman, P. Pook, and J. C. Watkins (1986) CPP, a new potent and selective NMDA antagonist. Depression of central neuron responses, affinity for $[3 \mathrm{H}] \mathrm{D}$-AP5 binding sites on brain membranes and anticonvulsant activity. Brain Res. 382: 169-173.

DelBuono, B. J., P. L. Williamson, and R. A. Schlegel (1988) Relation between the organization of spectrin and of membrane lipids in lymphocytes. J. Cell Biol. 106: 697-703.

Dunwiddie, T. V., and G. Lynch (1979) The relationship between extracellular calcium concentration and the induction of hippocampal long-term potentiation. Brain Res. 169: 103-110.

Fink, R. P., and L. Heimer (1967) Two methods for selective silver impregnation of degenerating axons and their synaptic endings in the central nervous system. Brain Res. 4: 369-374.

French, E. D., C. Aldinio, and R. Schwarcz (1982) Intrahippocampal kainic acid, seizures and local neuronal degeneration: Relationships assessed in unanesthetized rats. Neuroscience 7: 2525-2536.

Garthwaite, G., F. Hajos, and J. Garthwaite (1986) Ionic requirements for neurotoxic effects of excitatory amino acid analogues in rat cerebellar slices. Neuroscience 18: 437-447.

Gill, R., A. C. Foster, and G. N. Woodruff (1987) Systemic administration of MK-801 protects against ischaemia-induced hippocampal neurodegeneration in the gerbil. J. Neurosci. 7: 3343-3349.

Goldschmidt, R. B., and O. Steward (1980) Preferential neurotoxicity of colchicine for granule cells of the dentate gyrus of the adult rat. Proc. Natl. Acad. Sci. USA 77: 3047-3051.

Goodman, S. R., and I. S. Zagon (1986) The neural cell spectrin skeleton: A review. Am. J. Physiol. 250: C347-C360.

Gruenthal, M., D. R. Armstrong, B. Ault, and J. V. Nadler (1986) Comparison of seizures and brain lesions produced by intracerebroventricular kainic acid and bicuculline methiodide. Exp. Neurol. 93: $621-630$.

Hajos, F., G. Garthwaite, and I. Garthwaite (1986) Reversible and irreversible neuronal damage caused by excitatory amino acid analogues in rat cerebellar slices. Neuroscience 18: 417-436.

Harris, E. W., A. H. Ganong, and C. W. Cotman (1984) Long-term potentiation in the hippocampus involves activation of N-methyl-Daspartate receptors. Brain Res. 323: 132-137.

Harris, E. W., A. H. Ganong, D. T. Monaghan, J. C. Watkins, and C. W. Cotman (1986) Action of 3-(2-carboxypiperazin-4-yl)-propyl-1phosphonic acid (CPP): A new and highly potent antagonist of $\mathrm{N}$-methyl-D-aspartate receptors in the hippocampus. Brain Kes. 382: 174-177.

Hattori, T., and E. G. McGeer (1977) Fine structural changes in the rat striatum after local injections of kainic acid. Brain Res. 129: 174180.

Herndon, R. M., J. T. Coyle, and E. Addicks (1980) Ultrastructural analysis of kainic acid lesion to cerebellar cortex. Neuroscience 5: $1015-1026$.

Jahr, C. E., and C. F. Stevens (1987) Glutamate activates multiple single channel conductances in hippocampal neurones. Nature 325 : 522-525.

Kleinschmidt, A., M. F. Bear, and W. Singer (1987) Blockade of NMDA receptors disrupts experience-dependent plasticity of kitten striate cortex. Science 238: 355-358.

Leonard, J. P., and M. M. Salpeter (1982) Calcium-mediated myopathy at neuromuscular junctions of normal and dystrophic muscle. Exp. Neurol. 76: 121-138.

Levine, J., and M. Willard (1983) Redistribution of fodrin (a component of the cortical cytoplasm) accompanying capping of cell surface molecules. Proc. Natl. Acad. Sci. USA 80: 191-195.

Lynch, $G$., and M. Baudry (1984) The biochemistry of memory: A new and specific hypothesis. Science 224: 1057-1063.

Lynch, G., J. Larson, S. Kelso, G. Barrionuevo, and F. Schottler (1983) Intracellular injections of EGTA block the induction of hippocampal long-term potentiation. Nature 305: 719-721.

MacDermott, A. B., M. L. Mayer, G. L. Westbrook, S. J. Smith, and J. L. Barker (1986) NMDA-receptor activation increases cytoplasmic calcium concentration in cultured spinal cord neurones. Nature 321: 519-522.

Maragos, W. F., T. Greenamyre, J. B. Penney, Jr., and A. B. Young (1987) Glutamate dysfunction in Alzheimer's disease: An hypothesis. Trends Neurosci. 10:65-68.

Mattson, M. P., P. Dou, and S. B. Kater (1988) Outgrowth-regulating actions of glutamate in isolated hippocampal pyramidal neurons. $\mathbf{J}$. Neurosci. 8: 2087-2100.

Nadler, J. V., and D. A. Evenson (1983) Use of excitatory amino acids to make axon-sparing lesions of hypothalamus. Methods Enzymol. 103: 393-400.

Nadler, J. V., B. W. Perry, and C. W. Cotman (1978) Intraventricular kainic acid preferentially destroys hippocampal pyramidal cells. Nature 271: 676-677.

Nadler, J. V., B. W. Perry, C. Gentry, and C. W. Cotman (1980) Degeneration of hippocampal CA3 pyramidal cells induced by intraventricular kainic acid. J. Comp. Neurol. 192: 333-359.

Nadler, J. V., D. A. Evenson, and G. J. Cuthbertson (1981) Comparalive toxicity of kainic acid and other acidic annino acids toward rat hippocampal neurons. Neuroscience 6: 2505-2517.

Olney, J. W. (1987) Excitatory amino acids and neuropsychiatric disorders. In Excitatory Amino Acid 1 ransmission, 'T. P. Hicks, D. Lodge, and H. McLennan, eds., pp. 217-224, Liss, New York

Olney, J. W., T. Fuller, and T. DeGubareff (1979) Acute dendrotoxic changes in the hippocampus of kainate treated rats. Brain Res. 176: 91-100.

Price, M. T., J. W. Olney, L.. Samson, and J. Labruyere (1985) Calcium influx accompanies but does not cause excitotoxin-induced neuronal necrosis in retina. Brain Res. Bull. 14: 369-376.

Rothman, S. (1984) Synaptic release of excitatory amino acid neurotransmitter mediates axonic neuronal death. J. Neurosci. 4: 1884 1891.

Rothman, S. M. (1985) The neurotoxicity of excitatory amino acids is produced by passive chloride influx. J. Neurosci. 5: 1483-1489.

Rothman, S. M., J. H. Thurston, and R. E. Hauhart (1987) Delayed neurotoxicity of excitatory amino acids in vitro. Neuroscience 22: 471-480.

Schwarcz, R., A. C. Foster, E. D. French, W. O. Whetsell, and C. Kohler (1984) Excitotoxic models for neurodegenerative disorders. Life Sci. 35: $19-32$.

Siman, R., and J. C. Noszek (1988) Excitatory amino acids activate calpain I and induce structural protein breakdown in vivo. Neuron 1 : 279-287.

Siman, R., C. Gall, L. S. Perlmutter, C. Christian, M. Baudry, and G. Lynch (1985a) Distribution of calpain I, an enzyme associated with degenerative activity, in rat brain. Brain Res. 347: 399-403.

Siman, R., M. Baudry, and G. Lynch (1985b) Regulation of glutamate receptor binding by the cytoskeletal protein fodrin. Nature $313: 225-$ 228.

Siman, R., M. Ahdoot, and G. Lynch (1987) Ontogeny, compartmentation and turnover of spectrin isoforms in rat central neurons. J. Neurosci. 7: 55-64. 
Simon, R. P., J. H. Swan, T. Griffiths, and B. S. Meldrum (1984) Blockade of N-methyl-D-aspartate receptors may protect against ischemic damage in the brain. Science 226: 850-852.

Srinivasan, Y., L. Elmer, J. Davis, V. Bennett, and K. Angelides (1988) Ankyrin and spectrin associate with voltage-dependent sodium channels in brain. Nature 333: 177-180.

Weiss, J., M. P. Goldberg, and D. W. Choi (1986) Ketamine protects cultured neocortical neurons from hypoxic injury. Brain Res. 380: 186-190.

Wieloch, T. (1985) Hypoglycemia-induced neuronal damage prevented by an N-methyl-D-aspartate antagonist. Science 230: 681683.
Zaczek, R., and J. T. Coyle (1982) Excitatory amino acid analogues: Neurotoxicity and seizures. Neuropharmacology 21: 15-26.

Zaczek, R., J. Collins, and J. T. Coyle (1981) N-methyl-D-aspartic acid: A convulsant with weak neurotoxic properties. Neurosci. Lett. 24: $181-186$.

Zimmerman, U.-J. P., and W. W. Schlaepfer (1984) Calcium-activated neutral protease (CANP) in brain and other tissues. Prog. Neurobiol. 23: $63-78$. 